\title{
Tests on droplets and dust suppression characteristics with self- priming spray magnetization
}

\author{
Zhong-Fei MA, Hui-Feng HUANG, Xin-Yu XIONG, Dong-Hua XU
}

\author{
School of the Environment and Safety Engineering,Jiangsu University,Zhenjiang 212013,China \\ E-mail: zfma2006@163.com
}

KEYWORD: Self-priming spray; magnetization; droplets characteristics; dedusting characteristics

\begin{abstract}
To improve the effect of spray and dust reduction, self-priming spray magnetization dedusting was put forward. Moreover, some experiments on droplets and dedusting characteristics were carried out, such as traditional single-nozzle spray, self-priming non-magnetic spray, selfpriming spray with magnets on throat, self-priming spray with magnets on the diffusion tube, and self-priming spray with magnets both on the throat and diffusion tube respectively. The results showed that the SMD value of the self-priming spray was reduced by approximately $12 \%$ to $20 \%$ and dust reduction efficiency was increased by approximately $5 \%$ to $7 \%$ compared with the conventional single nozzle, while the SMD value of self-priming spray with magnets was reduced by approximately $10 \%$ to $15 \%$ and dust reduction efficiency was increased by approximately $6 \%$ to $15 \%$ compared with the self-priming spray without magnets, the SMD value of self-priming spray with magnets both on the throat and the diffusion tube was less than that of self-priming spray plus magnets on throat or diffusion tube alone, and the dust reduction efficiency was increased by approximately $5 \%$.
\end{abstract}

\section{INTRODUCTION}

Wet dust suppression is one of the most economic, simple and practical methods of dust suppression. It is applied in fields such as vehicle transportation, road construction, rock blasting of water conservancy and hydropower project, coal handling systems of power plant, tunnel construction, stock ground of metallurgical enterprise, concentrating mill, mine and other producing dust places,but the dust suppression effect of conventional single nozzle spray was limited. Improving dust suppression effect was researched on charged water spray, high-pressure spray, magnetized water spray, and pressure gas and water spray,which are limited in term of dust reduction efficiency and applicability ${ }^{[1-7]}$. Therefore, the trial tests on self-priming spray magnetization dust suppression were carried out on the basis of previous studies.

\section{PRINCIPLE OF DUST SUPPRESSION}

Self-priming spray was generated by the self-priming sprayer. The so-called self-priming sprayer was composed of a nozzle, throat, short diffusion tube, the suction chamber and other components, which could improve atomization and dedusting effect because ambient air can be inhaled when pressure water atomized by a nozzle ${ }^{[8-9]}$, as shown in Fig. 1.

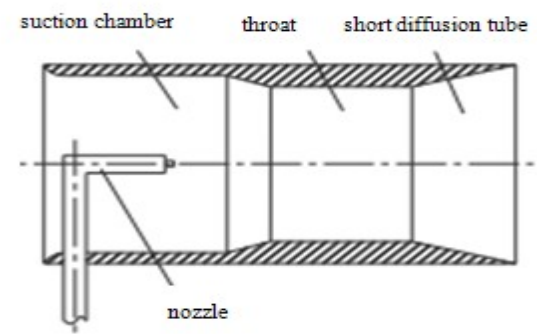

Fig. 1 The structure diagram of self-priming dust uppression sprayer

Self-priming spray magnetization dust suppression was the spray and dust reduction added magnetization structure on the throat or diffusion tube of self-priming sprayer, thus it was the combined effects of self-priming spray and magnetization spray.

The working principle was as follows: After going through the nozzle and producing high-speed jet, the high-pressure water was dispersed to form droplets which conducted an atomization. Then the 
droplets went into the vortex jet by entrainment of ambient air and generated negative pressure by momentum exchange with the suction air, which made ambient air drawn into the throat from the suction chamber and the external. At the same time, because of the smallest cross-section of the throat and the minimum static pressure, the droplets were broken into smaller sizes by colliding with ambient air. When the jet went into the diffusion tube, because of the lower gas velocity in the front, the surrounding gas went further into the jet, and the liquid-gas collision happened again and the unstable water-gas type bubbles were formed ${ }^{[10-12]}$. Meanwhile, the spray was magnetized by the magnetic structure, resulting a significant change in the structure and properties of spray water. Namely, the structure of the water spray changed from complex and long bond appearance to a short bond, the crystal structure became shorter, the bond angle of $\mathrm{H}_{2} \mathrm{O}$ changed, the hydrogen bonding was curved, a sudden increase and later softness in the hardness of the water, water conductivity and viscosity reduced, water droplets became thinner and smaller, the surface tension of water spray was reduced, and sorption capacity, solubility and permeability were increased. Then, after coming out from the diffusion tube, the spray spout in tiny droplets in magnetization and bubbles mixture, namely foam mixture form, and the mist was more uniform, thus improving dust suppression effect relying on increased inertial impaction, interception, brownian diffusion and aggregation.

\section{TEST METHODS}

\subsection{The structure of self-priming magnetized test spray}

The main component of self-priming magnetized test spray was nozzle, throat, suction chamber, magnets, conical concentrator device, mounting bracket, as shown in Fig. 2.

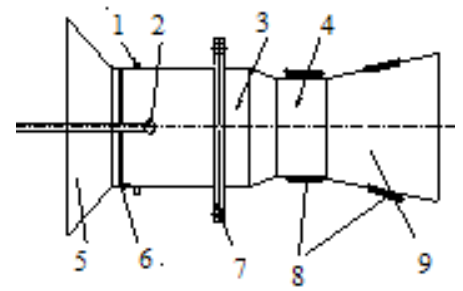

1-Static tube, 2-Nozzle, 3-Suction chamber, 4-Pipe,5-The wind collecting device, 6-

Mountingbracket,7-Flange, 8-Magnet, 9-Diffusion tube

Fig. 2 The structure diagram of test self-priming dust suppression sprayer

The device was selected with the following parameter: suction chamber diameter of $80 \mathrm{~mm}$, the suction chamber length of $115 \mathrm{~mm}$, throat diameter of $70 \mathrm{~mm}$, pipe length of $35 \mathrm{~mm}$, half-angle on the reducing pipe of $18^{\circ}$, diffusion angle on the diffusion tube of $45^{\circ}$; the corresponding length on the diffusion tube of $36 \mathrm{~mm}$, hose nozzle distance of $68 \mathrm{~mm}$, cone concentrator device diameter of $140 \mathrm{~mm}$, length of $30 \mathrm{~mm}$ and opening angle of $45^{\circ}$. The sizes and shapes of permanent ferrites used were shown in Fig. 3.

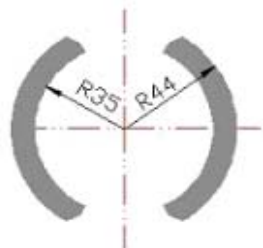

(a)

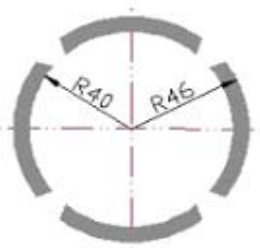

(b)

Fig. 3 Schematic of experimental magnets

Two ferrites with diameter of $35 \mathrm{~mm}$ and thickness of $9 \mathrm{~mm}$ were placed on the outer surface of the throat during the experiment, as shown in Fig. 3a.The shapes of the ferrites put in the water pipe of diameter of $19 \mathrm{~mm}$ are also shown in Fig. 3a. Four ferrites with the diameter of $40 \mathrm{~mm}$ and thickness of $6 \mathrm{~mm}$ were put on the outer surface of the diffusion tube during experiment, as shown in Fig. $3 b$.The role of the wind collecting device was to determine amount of air sucking. The role of the mounting bracket was to position the nozzle using the X-type swirl nozzle. Two static pressure measuring holes were opened at the bisection point of the pipe wall which was $15 \mathrm{~mm}$ away from the inlet section of the wind collecting device. A pipe was weld on each hole ensuring the 
connection part between the pipe and the test tube was as smooth as possible. The shell of the throat, suction chamber, diffusion tubes, and the conical concentrator was made from $1.2 \mathrm{~mm}$ thickness white metal, and flange was made from $1.5 \mathrm{~mm}$ thickness white metal, and static tube was copper tube by solder connection.

\subsection{Test System and Parametric measurement}

The test system was mainly composed of high pressure pump, water tank, self-priming magnetized sprayer, thermometer, hygrometer, water valve, water flow meter, dust generator and dust sampler as shown in Fig. 4. Dust concentrations were measured with weight method, the weight of filter membrane was weighed with the electronic balance. The particle size distribution was measured with laser particle analyzer, and the nozzle pressure was read by the pressure gauge on the high pressure pump.

Sauter mean diameter (SMD) was measured with Phase Doppler Particle Analyzer( PDPA), which mainly consisted of laser device, emitting lens, receiving lens, computer, pressure bottle and the precision pressure gauge. When the experiment about SMD was in progress, the spray was horizontally fixed on the bracket, and the set coordinate system was that along the center line of the spray was the transverse direction (Y-axis), while the direction of the moving direction of the transmitting probe was the longitudinal direction (Z-axis).

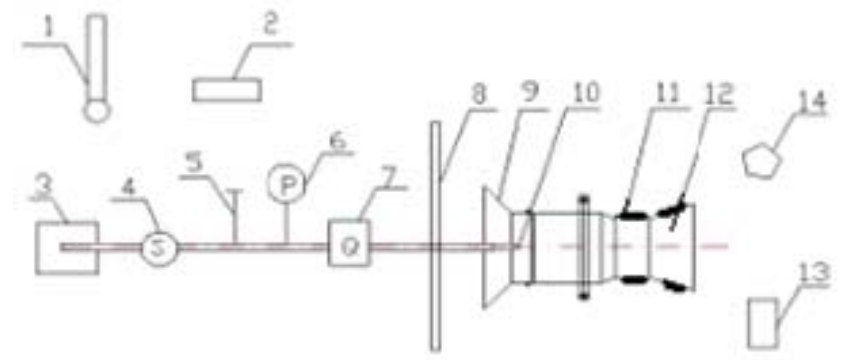

1-thermometer, 2-hygrometer, 3-tank, 4-high pressure water pump, 5-pressure regulating valve, 6-gauge, 7-flowmeter, 8-bracket, 9-the wind collecting device, 10-nozzle, 11-ferrite magnets, 12-jet pipe, 13- vibrating dust generator, 14-dust sampler

Fig.4 Test system

During the test, the water pressure was initially adjusted to $1 \mathrm{MPa}$, the automatic displacement mechanism of the PDPA was moved in the longitudinal direction to make the distance of the downstream longitudinal section of the spray (Z-axis) $0 \mathrm{~mm}, 15 \mathrm{~mm}, 30 \mathrm{~mm}, 45 \mathrm{~mm}, 60 \mathrm{~mm}, 75$ $\mathrm{mm}, 90 \mathrm{~mm}, 105 \mathrm{~mm}, 120 \mathrm{~mm}, 135 \mathrm{~mm}$ and $150 \mathrm{~mm}$, and the particle size distribution of the droplets was determined respectively. And then, water pressure was adjusted to $2 \mathrm{MPa}$ and $4 \mathrm{MPa}$, in turns, the SMD values were measured before and after magnetization and also at different magnetized location.

\section{RESULTS AND DISCUSSION}

\subsection{Droplets characteristics}

The main index of the droplets characteristics was SMD value. When the water pressure were $1 \mathrm{MPa}, 2 \mathrm{MPa}, 4 \mathrm{MPa}$ respectively, the determination data of SMD values are shown in Fig. 5, Fig. 6, Fig. 7, in the downstream section of the single nozzle spray, self-priming without magnets, selfpriming with magnets added on the throat, self-priming with magnets added on the diffusion tube, and self-priming with magnets added both on the throat and diffusion tube. 


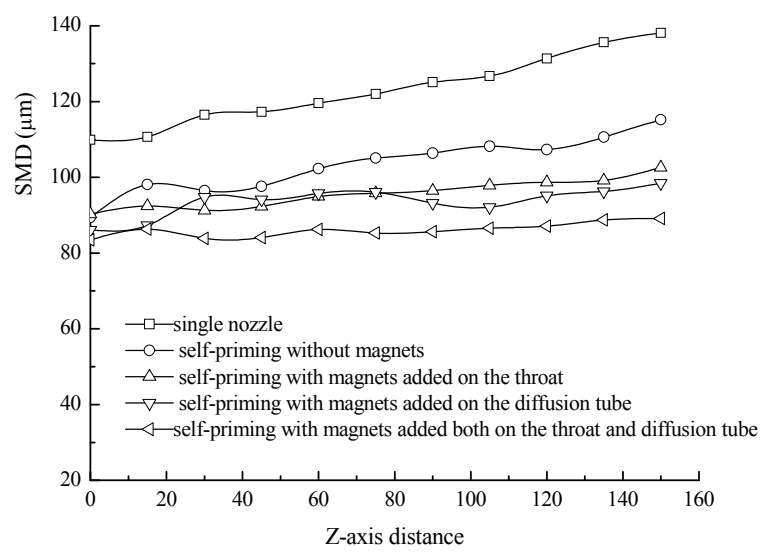

Fig.5 Distribution of SMD with water pressure 1MPa

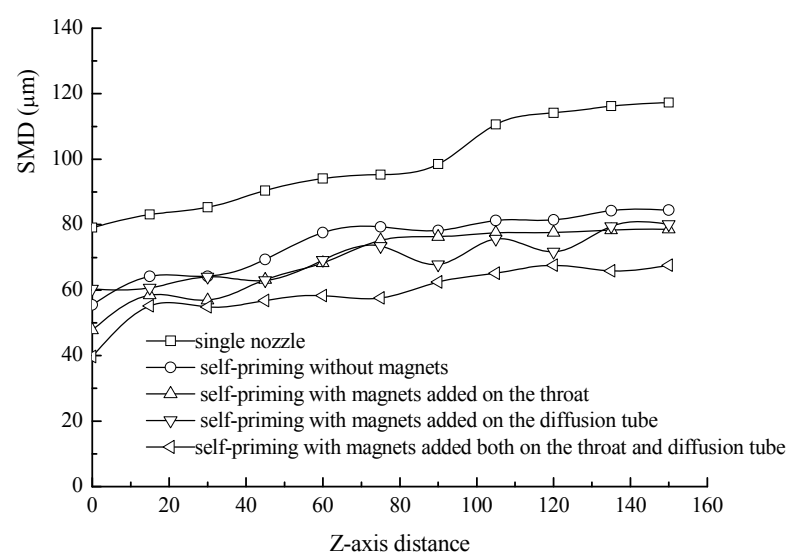

Fig.6 Distribution of SMD with water pressure $2 \mathrm{MPa}$

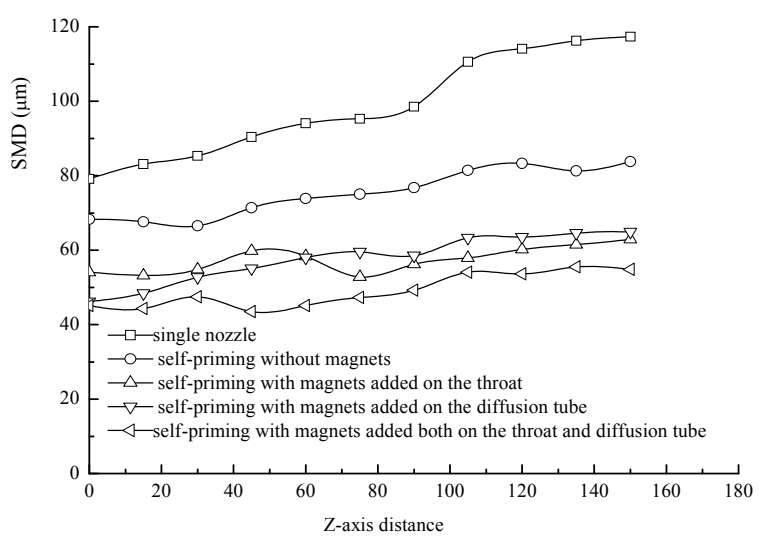

Fig.7 Distribution of SMD with water pressure 4Mpa

The followings can be seen:(1) The SMD value of the self-priming spray was reduced by approximately $12 \%$ to $20 \%$ compared with the conventional single nozzle, which indicated the better effect of self-priming spray without magnets because self-priming spray played a role in secondary atomization.(2)The SMD value of self-priming with magnet was reduced by approximately $10 \%$ to $15 \%$ compared with the self-priming without magnets, that is the better effect of self-priming magnetic spray because the crystal structure, surface tension and viscosity of water were momentarily changed.(3)The SMD value of the self-priming with magnets added both on the throat and diffusion tube was smaller than self-priming with magnets on the throat or 
diffusion tube alone, which illustrated that SMD value was related with magnetic intensity, and the greater the magnetic intensity, the smaller the SMD value. (4) There were fluctuations in SMD value changes of self-priming with magnets on the throat or diffusion tube alone, but there were little differences on the whole, which illustrated that there were little differences in self-priming with magnets added on the throat or diffusion tube alone.

\subsection{Dedusting characteristics}

During the test, the ambient temperature was $18.5^{\circ} \mathrm{C}$, relative humidity was $94 \%$, the air density was $1.20 \mathrm{~kg} / \mathrm{m}^{3}$, the test dust was intercepted by the bag filter in the power plant and then processed by the ball mill. Dust particle size distribution measured by CIS-50 laser particle size analyzer was shown in Table1. The dedusting test was conducted with the single nozzle spray, selfpriming without magnets, self-priming with magnets added on water pipe, self-priming with magnets added on the throat, self-priming with magnets added on the diffusion tube, and selfpriming with magnets added both on the throat and diffusion tube.

When the water pressure was $1 \mathrm{MPa}, 2 \mathrm{MPa}$ and $4 \mathrm{MPa}$ respectively, the measured results of the dust concentration and dedusting efficiency are shown in Fig. 8 and Fig. 9. Wherein, 1 of the abscissa in Fig. 8 represents the original dust concentration, and 2, 3, 4, 5, 6 of the abscissa in Fig. 8 and Fig.9 represent single nozzle spray, self-priming without magnets, self-priming with magnets added on water pipe, self-priming with magnets added on the throat, self-priming with magnets added on the diffusion tube, and self-priming with magnets added both on the throat and diffusion tube.

Table 1 Particle size distribution of dust

\begin{tabular}{cccccccc}
\hline $\begin{array}{c}\text { Particle size } \\
\text { interval } / \mu \mathrm{m}\end{array}$ & $<0.1$ & $0.1 \sim 0.5$ & $0.5 \sim 2$ & $2 \sim 5$ & $5 \sim 10$ & $10 \sim 20$ & $20 \sim 30$ \\
\hline percentage $\%$ & 0.23 & 12 & 20.1 & 15.8 & 10.7 & 15.5 & 5.67 \\
\hline
\end{tabular}

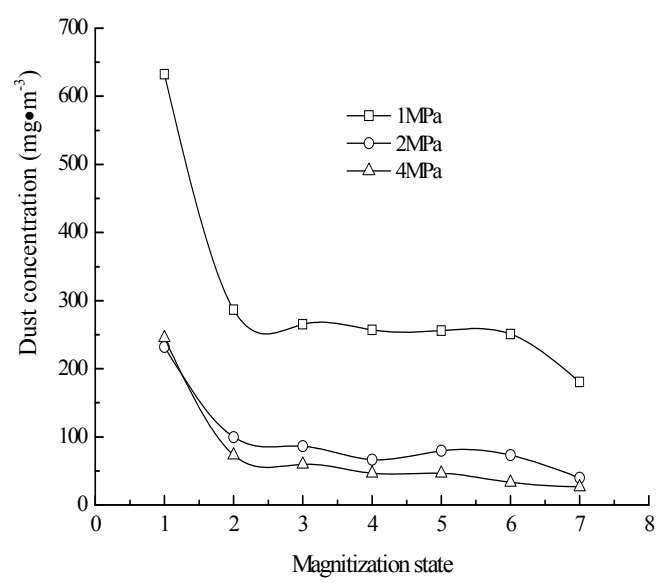

Fig.8 dust concentration in different states

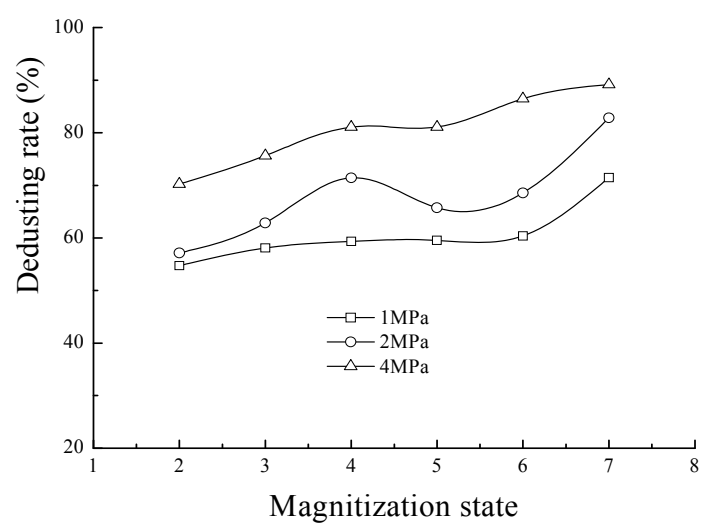

Fig.9 Dedusting efficiency in various states 


\section{CONCLUSIONS}

(1) The method of self-priming spray magnetization dedusting was used in the study, and its principle was deep analyzed. The work has theoretical significance and practical value on the improvement of wet dedusting effect.

(2) The experiments on droplet characteristics showed that SMD value of the self-priming spray was reduced by approximately $12 \%$ to $20 \%$ compared with the conventional single nozzle, and SMD value of self-priming with magnets was reduced by approximately $10 \%$ to $15 \%$ compared with the self-priming without magnets, and SMD value of the self-priming with magnets added both on the throat and diffusion tube was less than that of self-priming with magnets on the throat or diffusion tube alone. That is that the SMD value was related with magnetic intensity, and the greater the magnetic intensity, the less the SMD value, and there were little differences in SMD value of self-priming with magnets added on the throat or diffusion tube alone.

(3) The experiment on dedusting characteristics showed that the dedusting efficiency of selfpriming spray was increased by approximately 5\% to $7 \%$ compared with the conventional single nozzle, and the dedusting efficiency of self-priming with magnets was increased by approximately $6 \%$ to $15 \%$ compared with the self-priming without magnets. The dedusting efficiency of the selfpriming with magnets added both on the throat and diffusion tube was greater than self-priming with magnets on the pipe or throat or diffusion tube alone, and the dedusting efficiency had little difference on the whole between the self-priming with magnets on the water pipe and the throat alone, while the dedusting efficiency of self-priming with magnets added on the diffusion tube was increased by approximately 5\% compared with self-priming with magnets added on the water pipe or the throat.

\section{ACKNOWLEDGMENTS}

The thesis was subsidized by 2016' graduate education innovation project (SJZZ16_0191) in jiangsu province .

\section{REFERENCES}

[1] Zhongfei Ma,.Industrial Ventilation and Dust control. In: Industrial technology. China labor safeguard press, Beijing. 2009, pp.168--173

[2]Andrzej Krupa, Anatol Jaworek, Arkadiusz T,. Charged spray generation for gas cleaning applications.Journal of electrostatics, 71(06),2013: 260-264

[3] Xiaoying Wang, Jianlong Wen,.Study on Charged spray characteristics of two-fluid nozzle. In: International Conference on New Technology of Agricultural Engineering. Zibo, Shandong. 27--29 May.2011:58--61.

[4] Xie J L, Gan ZW, Duan F. Characterization of spray atomization and heat transfer of pressure swirl nozzles. INTERNATIONAL JOURNAL OF THERMAL SCIENCES, 68(06),2013:94-102.

[5] Aiping Zhou, Desheng Wang.Study on the intake wind performance experiment of the pipe with high pressure spray. Applied Mechanics and Materials. Manufacturing and Engineering Developments. 273,2013:115-118

[6] Xingguo Wang, Li Li, Yuzhou Zheng, Experimental Study on Sprayed Concrete Mixed with Magnetized Water. Applied Mechanics and Materials. 2011: 3524-3528

[7] Yongwen Chen, Wuxue Han, Xiaohong Li, et al., Analysis on test of windwater spraying dropdust of fully mechanized caving workface in Shigejie coal mine. Coal, 9(5),2000..pp 2728.

[8] Ajmal Shah, Imran Rafiq Chughtai, Mansoor Hameed Inayat. Experimental study of the characteristics of steam jet pump and effect of mixing section length on direct-contact condensation.International Journal of Heat and Mass Transfer, 58(03),2013: 62--69. 
[9] Lijun Yang, Qingfei Fu, Wei Zhang, Ming-long Du, Ming-xi Tong,. Spray Characteristics of Gelled Propellants in Novel Impinging Jet Injector. Journal of Propulsion and Power, 29(01) 2013,pp104--113.

[10] Zhongfei Ma, Yu-Xiang Zhang, Xiuli Yang, Zhilin Ge, Experiment on dust suppression performance of self-priming sprayer. Journal of drainage and irrigation machinery engineering.30(01), 2012:97-101

[11] Zhongfei Ma, Zhen Zhang, Zhilin Ge, Numerical simulation on self-priming spray and conventional spray characteristics. Applied Mechanics and Materials. 261-262,2012:948-953

[12] Zhongfei Ma, Penggang Jin, Feng Zhao.Test on characteristics of the high-pressure secondary rotary water jet ventilation. Advanced Materials Research. 594—597,2012: 2167-2170 